\title{
How to Read a Paper: The Basics of Evidence Based Medicine, 2nd ed.
}

By Trisha Greenhalgh. 222 pp. London, BMJ Books; 2001. 16.95 GBP (Great British Pound). ISBN: 0-7279-1578-9

The implementation of evidence based medicine has dramatically changed the landscape in how clinicians manage their patients. The initial skepticism has much faded and it is now generally embraced by the whole medical community. However, evidence based medicine involves concepts and mathematics that may not be familiar to not only medical students but to expert clinicians as well. Therefore, it may be difficult to critically read a paper and decide whether the "evidence" is really there. Because clinicians often read papers to not only gain knowledge but to alter their medical practice, it becomes crucial that they not just read but "to read the right papers at the right time." In this context, this book is an excellent introduction to the basics of evidence based medicine and teaches one to appraise the pa- per that he or she is reading as the "right paper." It is written in easy to understand language with every day examples that augments the reader's understanding, yet it is concise enough to prevent the reader from drifting away. Text boxes that either summarize concepts or explain terms are also a welcome. This book is an excellent source for befriending evidence based medicine especially to those who have not done so during medical school.

Jang Hwan Kim Department of Urology, Yonsei University College of Medicine E-mail: jkim@yuhs.ac 